\title{
Introduction to Special Issue: Embodied Interactive Robots
}

Published online: 10 June 2021

(c) The Author(s) 2021

The current COVID-19 pandemic situation has put attention into social robotics stakes and challenges, namely, in their ability to seizure the problem of human virus transmission, by transferring some risky labors to robotic systems. Examples come from different companies, like disinfecting ultraviolet (UV) rovers and ground drones for hospitals, or even beaming videos to connect patients to their relatives illustrated by some telepresence robots. Many non-value-added nursing procedures could have been achieved by an assistive robot, lowering unnecessary virus exposure risks. There are more examples in other fields, like flagging patients with suspected pneumonia during their hospital admission, etc. It appears clearly that social robotics is one of the most complex and noble robotic perspectives, and the expectations are extremely high: improving quality of human life in all sectors through various shades of human-robot assistance and partnerships. The 2017 edition of ICSR fostered several rich exchanges and discussions on the development of robotic solutions for better embodiments, and interactive behaviors that enable social robots to have an impact on the degree of personalized companionship with humans. The following are selected papers, representative of the issues that were convered.

The first paper investigates the influence of using a telepresence robot in a private facility on the resident, family members, and care workers. Different trials with a telepresence robot Double were conducted at a private facility and a public facility. Their findings highlight important ethical guidelines pertaining using telepresence in residential care. This work is even more timely with respect to the current pandemic situation.

In the second paper, insights about designing a storytelling robot are presented. Three perception studies were conducted with different modalities. The results provided

This special issue gathers selected papers from the Ninth International Conference on Social Robotics (ICSR 2017) that was held in Tsukuba, Japan for the first time since its establishment.

The theme of this edition is embodied interactive robots. guidance for the modeling and evaluation of storytelling robots. Such work has implications in social educational robots.

In the third paper, a mobile platform is developed to help patients with lower-limb sensory motor paralysis during walk rehabilitation. The experimental results indicate the adaptability of the platform to the walking pattern of the participants and the social improvements it gave them and is of high interest in customized and efficient rehabilitation.

The fourth article investigates how the perceived gender controlled by audio and visual stimuli affect the perceived effects in a robot's hug. The study used an integration of a robot and a virtual reality to prepare the agents. The results demonstrate that agents' genders affect the robot's hug impressions.

The fifth article investigates the perceived social effects of changing some dimensions of a robot body. The results show different participants' perceived impressions when dealing with a robot with a female body shape as compared to one with a male body shape. Gender equity and minority visibility are now an important stake in our modern society and this work could contribute on promoting it from the social robots design.

The sixth paper presents the development history of Kaspar. The article describes the rationale behind the changes to the Kaspar platform since its creation. The development history of Kaspar's design should provide useful insights for future roboticists in social robotics. Autism spectrum disorder is universal phenomenon not very well understood and where robotic could play an interesting role in the diagnosis and targeted care and educational and perspectives.

The seventh article conducts an empirical test to investigates the acceptability and performance of a robot to promote in fashion industry. The results show the potential of using the robot to attract the attention of customers toward a showcase in a store. It hence might offer and alternative perspectives to current static manikins.

The final article examines the evaluation of a humanoid robot by users based on different eye contact experiments. The work provides important design insights and 
considerations about eye contact in human-robot interaction, which is or prime importance in social robot-human interactions at large.
Publisher's Note Springer Nature remains neutral with regard to jurisdictional claims in published maps and institutional affiliations. 